\title{
Gelfand pairs associated with finite Heisenberg groups
}

\author{
Chal Benson and Gail Ratcliff \\ Dept of Mathematics, East Carolina University, Greenville, NC 27858 \\ bensonf@ecu.edu, ratcliffg@ecu.edu
}

A topological group $G$ together with a compact subgroup $K$ are said to form a Gelfand pair if the set $L^{1}(K \backslash G / K)$ of $K$-bi-invariant integrable functions on $G$ is a commutative algebra under convolution. The situation where $G$ and $K$ are Lie groups has been the focus of extensive and ongoing investigation. Riemannian symmetric spaces $G / K$ furnish the most widely studied and best understood examples. ([Hel84] is a standard reference.) Apart from these, key examples arise as semi-direct products $G=K \ltimes N$, of compact Lie groups $K$ with two-step nilpotent Lie groups $N$. Such pairs are the focus of [BJR90], [Vin03] and [Yak06], among other works. There are many examples where $N=H_{n}(\mathbb{R})$, a (real) Heisenberg group, and $K$ is a subgroup of the unitary group $U(n)$.

Gelfand pairs also arise in connection with analysis on finite groups, but, to our knowledge, have been studied less extensively. Known examples include the symmetric group modulo the hyperoctahedral group [Mac] and finite analogues of the hyperbolic plane [SA87, Ter99]. In this paper we introduce a family of Gelfand pairs associated with finite Heisenberg groups. They provide finite analogues for the Gelfand pairs associated with $H_{n}(\mathbb{R})$. Our examples appear elsewhere, in the literature on the oscillator representation, but their relevance to the study of Gelfand pairs has not, however, been previously emphasized.

\section{Preliminaries}

To begin we must establish notation and recall some ideas concerning the representation theory for Heisenberg groups over finite fields. For a finite set $S$, the symbol $\mathbb{C}[S]$ will denote the set of all $\mathbb{C}$-valued functions on $S$. This is a complex vector space of dimension $|S|$ which carries a positive-definite hermitian inner product

$$
\langle f, g\rangle_{S}=\frac{1}{|S|} \sum_{x \in S} f(x) \overline{g(x)} .
$$




\subsection{Heisenberg groups}

Let $\mathbb{F}$ be a field of odd characteristic. The polarized Heisenberg group $H_{n}(\mathbb{F})$ is the set

$$
H_{n}(\mathbb{F})=\mathbb{F}^{n} \times \mathbb{F}^{n} \times \mathbb{F}
$$

with product

$$
(\mathbf{x}, \mathbf{y}, t)\left(\mathbf{x}^{\prime}, \mathbf{y}^{\prime}, t^{\prime}\right)=\left(\mathbf{x}+\mathbf{x}^{\prime}, \mathbf{y}+\mathbf{y}^{\prime}, t+t^{\prime}+\frac{1}{2}\left(\mathbf{x} \cdot \mathbf{y}^{\prime}-\mathbf{y} \cdot \mathbf{x}^{\prime}\right)\right) .
$$

Inclusion of the factor $1 / 2$ is motivated by the use of exponential coordinates in connection with the real Heisenberg group $H_{n}(\mathbb{R})$. (See [Fol89].) Some authors omit this factor but the resulting group is isomorphic with that defined here via the mapping $(\mathbf{x}, \mathbf{y}, t) \mapsto(\mathbf{x}, \mathbf{y}, 2 t)$.

An alternate notation is useful in connection with certain examples and constructions. We write

$$
\mathcal{W}=\mathcal{W}_{n}=\mathbb{F}^{n} \times \mathbb{F}^{n},
$$

so that $H_{n}(\mathbb{F})=\mathcal{W} \times \mathbb{F}$ and Equation (1) becomes

$$
(\mathbf{z}, t)\left(\mathbf{z}^{\prime}, t^{\prime}\right)=\left(\mathbf{z}+\mathbf{z}^{\prime}, t+t^{\prime}+2^{-1}\left[\mathbf{z}, \mathbf{z}^{\prime}\right]\right)
$$

where $\left[\mathbf{z}, \mathbf{z}^{\prime}\right]$ denotes the usual symplectic form on $\mathcal{W}$, namely

$$
\left[\mathbf{z}, \mathbf{z}^{\prime}\right]=\left[(\mathbf{x}, \mathbf{y}),\left(\mathbf{x}^{\prime}, \mathbf{y}^{\prime}\right)\right]=\mathbf{x} \cdot \mathbf{y}^{\prime}-\mathbf{y} \cdot \mathbf{x}^{\prime} .
$$

More generally, for any finite dimensional symplectic vector space $(\mathcal{W},[\cdot, \cdot])$ over $\mathbb{F}$ we let

$$
H_{\mathcal{W}}=\mathcal{W} \times \mathbb{F} \text { with product given by }(2) \text {. }
$$

\subsection{Unitary dual of $H_{n}\left(\mathbb{F}_{q}\right)$}

Throughout this paper we take

$$
\mathbb{F}=\mathbb{F}_{q},
$$

the finite field with $q$ elements where $q=p^{m}$ for some odd prime $p$. The field $\mathbb{F}$ is an extension of its prime field $\mathbb{Z}_{p}=\mathbb{Z} /(p \mathbb{Z})$. The characters on $\mathbb{F}$ are

$$
\widehat{\mathbb{F}}=\left\{\psi_{a}: a \in \mathbb{F}\right\}
$$

where

$$
\psi_{a}(t)=\exp \left(\frac{2 \pi i}{p} \operatorname{Tr}_{\mathbb{F} / \mathbb{Z}_{p}}(a t)\right)
$$

and

$$
\operatorname{Tr}_{\mathbb{F} / \mathbb{Z}_{p}}: \mathbb{F} \rightarrow \mathbb{Z}_{p}
$$


is the trace map for the field extension $\mathbb{F} / \mathbb{Z}_{p}$. Explicitly one can write

$$
\operatorname{Tr}_{\mathbb{F} / \mathbb{Z}_{p}}(t)=t+t^{p}+t^{p^{2}}+\cdots+t^{p^{m-1}}
$$

(See Chapter 2 in [LN].) The basic identity

$$
\sum_{t \in \mathbb{F}} \psi_{a}(t)=\left\{\begin{array}{l}
q \text { if } a=0 \\
0 \text { if } a \neq 0
\end{array}\right\}=q \delta_{a, 0} .
$$

shows that the characters are pair-wise orthogonal unit vectors in $\mathbb{C}[\mathbb{F}]$.

The mappings

$$
\Psi_{\mathbf{a}, \mathbf{b}}: H_{n}(\mathbb{F}) \rightarrow \mathbb{T}, \quad \Psi_{\mathbf{a}, \mathbf{b}}(\mathbf{x}, \mathbf{y}, t)=\prod_{i=1}^{n} \psi_{a_{i}}\left(x_{i}\right) \prod_{j=1}^{n} \psi_{b_{j}}\left(y_{j}\right)
$$

for $\mathbf{a}, \mathbf{b} \in \mathbb{F}^{n}$ give $q^{2 n}$ distinct 1-dimensional representations of $H_{n}(\mathbb{F})$. One can verify, moreover, that for $\lambda \in \mathbb{F}^{\times}$, the formula

$$
\pi_{\lambda}(\mathbf{x}, \mathbf{y}, t) f(\mathbf{u})=\psi_{\lambda}\left(t+\mathbf{y} \cdot \mathbf{u}+2^{-1} \mathbf{x} \cdot \mathbf{y}\right) f(\mathbf{u}+\mathbf{x})
$$

defines a unitary representation (analogous to the Schrödinger model in the real case) of $H_{n}(\mathbb{F})$ in the the inner product space $\mathbb{C}\left[\mathbb{F}^{n}\right]$. The trace character $\chi_{\lambda}(\mathbf{x}, \mathbf{y}, t)=\operatorname{tr}\left(\pi_{\lambda}(\mathbf{x}, \mathbf{y}, t)\right)$ for $\pi_{\lambda}$ is

$$
\chi_{\lambda}(\mathbf{x}, \mathbf{y}, t)=q^{n} \delta_{\mathbf{x}, 0} \delta_{\mathbf{y}, 0} \psi_{\lambda}(t),
$$

which yields

$$
\left\langle\chi_{\lambda}, \chi_{\lambda^{\prime}}\right\rangle_{H_{n}(\mathbb{F})}=\left\langle\psi_{\lambda}, \psi_{\lambda^{\prime}}\right\rangle_{\mathbb{F}}=\delta_{\lambda, \lambda^{\prime}}
$$

in view of orthogonality for the characters of $\mathbb{F}$. It follows that the representations $\left\{\pi_{\lambda}: \lambda \in \mathbb{F}^{\times}\right\}$are inequivalent and irreducible.

Summing the squares of the dimensions for the representations (7) and (8) gives

$$
q^{2 n} \times 1^{2}+(q-1) \times\left(q^{n}\right)^{2}=q^{2 n+1}=\left|H_{n}(\mathbb{F})\right| .
$$

Thus (7) and (8) exhaust the unitary dual of $H_{n}(\mathbb{F})$ :

$$
\widehat{H_{n}(\mathbb{F})}=\left\{\Psi_{\mathbf{a}, \mathbf{b}}: \mathbf{a}, \mathbf{b} \in \mathbb{F}^{n}\right\} \uplus\left\{\pi_{\lambda}: \lambda \in \mathbb{F}^{\times}\right\} .
$$

On the center of $H_{n}(\mathbb{F})$ we have $\pi_{\lambda}(0,0, t)=\psi_{\lambda}(t) I_{\mathbb{C}\left[\mathbb{F}^{n}\right]}$. So the $q^{n}$-dimensional irreducible representations are determined by their central characters. This proves:

Theorem 1.1 (Stone-von Neumann Theorem) Let $\lambda \in \mathbb{F}^{\times}$and $\beta$ : $H_{n}(F) \rightarrow U(V)$ be an irreducible unitary representation with central character $\psi_{\lambda}$. (That is, $\beta(0,0, t)=\psi_{\lambda}(t) I_{V}$.) Then $\beta$ is unitarily equivalent to the Schrödinger representation $\pi_{\lambda}$ defined by Equation 8. 


\subsection{Oscillator representation}

The symplectic group for $\left(\mathcal{W}=\mathbb{F}^{n} \times \mathbb{F}^{n},[\cdot, \cdot]\right)$,

$$
S p(n, \mathbb{F})=\left\{g \in G L(2 n, \mathbb{F}):\left[g \mathbf{z}, g \mathbf{z}^{\prime}\right]=\left[\mathbf{z}, \mathbf{z}^{\prime}\right]\right\},
$$

acts by automorphisms on $H_{n}(\mathbb{F})$ via

$$
g \cdot(\mathbf{z}, t)=(g \mathbf{z}, t) .
$$

Fix $\lambda \in \mathbb{F}^{\times}$. For given $g \in S p(n, \mathbb{F})$,

$$
(\mathbf{z}, t) \mapsto \pi_{\lambda} \circ g(\mathbf{z}, t)=\pi_{\lambda}(g \mathbf{z}, t)
$$

is an irreducible representation with central character $\psi_{\lambda}$. The Stone-von Neumann Theorem ensures that $\pi_{\lambda} \circ g$ is unitarily equivalent to $\pi_{\lambda}$. Thus there is a unitary operator $\omega_{\lambda}(g)$ on $\mathbb{C}\left[\mathbb{F}^{n}\right]$ satisfying

$$
\pi_{\lambda}(g \mathbf{z}, t)=\omega_{\lambda}(g) \pi_{\lambda}(\mathbf{z}, t) \omega_{\lambda}(g)^{-1} .
$$

Schur's Lemma shows that (10) defines $\omega_{\lambda}(g)$ up to a multiplicative scalar of modulus one. In the context of finite fields, there is a systematic choice of scalars for which

$$
\omega_{\lambda}: S p(n, \mathbb{F}) \rightarrow U\left(\mathbb{C}\left[\mathbb{F}^{n}\right]\right)
$$

is a representation of the group $S p(n, \mathbb{F})$. In the literature, $\omega_{\lambda}$ is variously called the oscillator, metaplectic, or Weil-Segal-Shale representation. It is known that $S p(n, \mathbb{F})$ coincides with its commutator subgroup provided $n>1$ or $q>3$. Thus (10) completely determines the representation $\omega_{\lambda}$, except when $n=1$ and $q=3$. (See [How].)

The contragredient representation $\pi_{\lambda}^{*}$ for $\pi_{\lambda}$ has central character $\psi_{-\lambda}$. Thus $\pi_{\lambda}^{*}$ is unitarily equivalent to $\pi_{-\lambda}$, by the Stone-von Neumann Theorem. Moreover the contragredient $\omega_{\lambda}^{*}$ of the oscillator representation satisfies $\pi_{\lambda}^{*}(g \mathbf{z}, t)=\omega_{\lambda}^{*}(g) \pi_{\lambda}^{*}(\mathbf{z}, t) \omega_{\lambda}^{*}(g)^{-1}$. It follows that

$$
\omega_{\lambda}^{*} \text { is unitarily equivalent to } \omega_{-\lambda} \text {. }
$$

There are, in fact, just two distinct oscillator representations $\omega_{\lambda}$, up to unitary equivalence. Indeed

Proposition 1.2 (See [How], [Neu02]) For $\lambda, \lambda^{\prime} \in \mathbb{F}^{\times}$one has $\omega_{\lambda}^{\prime} \simeq \omega_{\lambda}$ if and only if $\lambda^{\prime} / \lambda$ is a square in $\mathbb{F}^{\times}$.

The oscillator representation can be rendered explicitly, at least on a set of generators for $S p(n, \mathbb{F})$. The formulas are given below in Theorem 1.3. Writing $(2 n) \times(2 n)$-matrices in block form,

$$
g=\left[\begin{array}{l|l}
A & B \\
\hline C & D
\end{array}\right] \quad(A, B, C, D \text { of size } n \times n),
$$


one has

$$
g \in S p(n, \mathbb{F}) \Longleftrightarrow\left\{A^{t} C=C^{t} A, \quad B^{t} D=D^{t} B, \quad A^{t} D-C^{t} B=I\right\} .
$$

The group $S p(n, \mathbb{F})$ is generated by the subset

$$
\left\{A_{\text {diag }}: A \in G L(n, \mathbb{F})\right\} \cup\left\{C_{\text {lower }}: C^{t}=C\right\} \cup\{J\}
$$

where

$$
A_{\text {diag }}=\left[\begin{array}{c|c}
A & 0 \\
\hline 0 & \left(A^{t}\right)^{-1}
\end{array}\right], \quad C_{\text {lower }}=\left[\begin{array}{c|c}
I \mid 0 \\
\hline C \mid I
\end{array}\right], \quad J=\left[\begin{array}{c|c}
0 \mid I \\
\hline-I \mid 0
\end{array}\right]
$$

Theorem 1.3 (See $[\mathbf{N e u 0 2}])$ For $\lambda \in \mathbb{F}_{q}^{\times}\left(q=p^{m}\right)$ the oscillator representation

$$
\omega_{\lambda}: S p(n, \mathbb{F}) \rightarrow U\left(\mathbb{C}\left[\mathbb{F}^{n}\right]\right)
$$

is given on the generators (12) for $S p(n, \mathbb{F})$ as follows.

- $\omega_{\lambda}\left(A_{\text {diag }}\right) f(\mathbf{u})=\operatorname{sgn}(\operatorname{det} A) f\left(A^{-1} \mathbf{u}\right)$ where

$$
\operatorname{sgn}(t)=\left\{\begin{array}{ll}
+1 & \text { if } t \text { is a square in } \mathbb{F} \\
-1 & \text { otherwise }
\end{array} .\right.
$$

- $\omega_{\lambda}\left(C_{\text {lower }}\right) f(\mathbf{u})=\psi_{\lambda}\left(-\frac{1}{2} \mathbf{u}^{t} C \mathbf{u}\right) f(\mathbf{u})$.

- $\omega_{\lambda}(J) f(\mathbf{u})=(-1)^{n(m+1)}(-i)^{n m(p-1) / 2} \operatorname{sgn}(\lambda) \overline{\mathcal{F}}_{\lambda} f(\mathbf{u})$ where

$$
\overline{\mathcal{F}}_{\lambda} f(\mathbf{u})=\frac{1}{\sqrt{q^{n}}} \sum_{\mathbf{x} \in \mathbb{F}^{n}} f(\mathbf{x}) \psi_{\lambda}(\mathbf{x} \cdot \mathbf{u}) .
$$

Note that $\overline{\mathcal{F}}_{\lambda} f$ is a $\lambda$-weighted variant of the ( $n$-dimensional) inverse discrete Fourier transform (DFT).

\section{The group algebra $\mathbb{C}\left[H_{n}\left(\mathbb{F}_{q}\right)\right]$ and Gelfand pairs}

Let $H$ denote the Heisenberg group $H=H_{n}(\mathbb{F})=\mathcal{W} \times \mathbb{F}$ where, as before,

$$
\mathcal{W}=\mathbb{F}^{n} \times \mathbb{F}^{n}, \quad \mathbb{F}=\mathbb{F}_{q}, \quad q=p^{m}, \quad p \text { an odd prime. }
$$

The convolution product on $\mathbb{C}[H]$ is

$$
\begin{aligned}
(f \star g)(\mathbf{z}, t) & =\sum_{\left(\mathbf{z}^{\prime}, t^{\prime}\right) \in H} f\left((\mathbf{z}, t)\left(\mathbf{z}^{\prime}, t^{\prime}\right)^{-1}\right) g\left(\mathbf{z}^{\prime}, t^{\prime}\right) \\
& =\sum_{\left(\mathbf{z}^{\prime}, t^{\prime}\right) \in H} f\left(\mathbf{z}-\mathbf{z}^{\prime}, t-t^{\prime}-2^{-1}\left[\mathbf{z}, \mathbf{z}^{\prime}\right]\right) g\left(\mathbf{z}^{\prime}, t^{\prime}\right) .
\end{aligned}
$$




\subsection{Twisted convolution on $\mathbb{C}[\mathcal{W}]$}

Twisted convolution is well-known in connection with analysis on the real Heisenberg group $H_{n}(\mathbb{R})$. (See [Fol89].) Here we require its discrete analog.

Definition 2.1 For $f \in \mathbb{C}[H]$ and $a \in \mathbb{F}$ define $f_{a} \in \mathbb{C}[\mathcal{W}]$ via

$$
f_{a}(\mathbf{z})=\frac{1}{\sqrt{q}} \sum_{t \in \mathbb{F}} f(\mathbf{z}, t) \psi_{a}(t) .
$$

For fixed $\mathbf{z} \in \mathcal{W}, f_{a}(\mathbf{z})$ is the (one-dimensional) inverse discrete Fourier transform of $t \mapsto f(\mathbf{z}, t)$ evaluated at $a$. The Fourier inversion formula yields

$$
f(\mathbf{z}, t)=\frac{1}{\sqrt{q}} \sum_{a \in \mathbb{F}} f_{a}(\mathbf{z}) \psi_{a}(-t) .
$$

In particular, a function $f \in \mathbb{C}[H]$ is completely determined by $\left\{f_{a}: a \in \mathbb{F}\right\}$. So for given $f, f^{\prime} \in \mathbb{C}[H]$,

$$
f=f^{\prime} \Longleftrightarrow f_{a}=f_{a}^{\prime} \text { for all } a \in \mathbb{F} .
$$

Definition 2.2 For functions $f, g \in \mathbb{C}[\mathcal{W}]$ and given $a \in \mathbb{F}$ we define the twisted convolution $f \natural_{a} g \in \mathbb{C}[\mathcal{W}]$ via

$$
\left(f \natural_{a} g\right)(\mathbf{z})=\sum_{\mathbf{w} \in \mathcal{W}} f(\mathbf{z}-\mathbf{w}) g(\mathbf{w}) \psi_{a}\left(\frac{1}{2}[\mathbf{z}, \mathbf{w}]\right) .
$$

A straightforward calculation using (13) and (6) yields the following.

Lemma 2.3 For $f, g \in \mathbb{C}[H]$ and $a \in \mathbb{F}$ one has

$$
(f \star g)_{a}=\sqrt{q} f_{a} \natural_{a} g_{a} .
$$

\section{2 $K$-invariant functions on $H$ and $\mathcal{W}$}

The symplectic group $S p(n, \mathbb{F})$ acts on $\mathbb{C}[\mathcal{W}]$ and $\mathbb{C}[H]$ via

$$
k \cdot f(\mathbf{z})=f\left(k^{-1} \mathbf{z}\right) \quad \text { and } \quad k \cdot f(\mathbf{z}, t)=f\left(k^{-1} \cdot(\mathbf{z}, t)\right)=f\left(k^{-1} z, t\right) .
$$

For subgroups $K$ of $S p(n, \mathbb{F})$ we let $\mathbb{C}[\mathcal{W}]^{K}$ and $\mathbb{C}[H]^{K}$ denote the sets of $K$-fixed elements in $\mathbb{C}[\mathcal{W}]$ and $\mathbb{C}[H]$ respectively. These are easily seen to be subalgebras of $\mathbb{C}[\mathcal{W}]$ and $\mathbb{C}[H]$ with respect to the convolutions $\natural_{a}$ and $\star$.

Definition 2.4 Given a subgroup $K$ of $S p(n, \mathbb{F})$, we say that $(K, H)$ is a Gelfand pair when $\mathbb{C}[H]^{K}$ is a commutative algebra under convolution.

Remark 2.5 One can identify $\mathbb{C}[H]^{K}$ with the algebra $\mathbb{C}[K \backslash G / K]$ of $K$-biinvariant functions on the semidirect product $G=K \ltimes H$. So $(G, K)$ is a Gelfand pair in the traditional sense when Definition 2.4 applies. 
Proposition 2.6 Let $K$ be a subgroup of $S p(n, \mathbb{F})$. Then $(K, H)$ is a Gelfand pair if and only if $h h^{\prime} \in\left(K h^{\prime}\right)(K h)$ for all $h, h^{\prime} \in H$.

Proof. This result is the discrete analog of Theorem 1.12 in [BJR90].

Proposition 2.7 Let $K$ be a subgroup of $S p(n, \mathbb{F})$. Then $(K, H)$ is a Gelfand pair if and only if $\left(\mathbb{C}[\mathcal{W}]^{K}, \natural_{\lambda}\right)$ is commutative for all $\lambda \in \mathbb{F}^{\times}$.

Proof. First note that $\left(\mathbb{C}[\mathcal{W}]^{K}, \natural_{0}\right)$ is, in any case, commutative since $\bigsqcup_{0}$ is the standard (untwisted) convolution on $\mathbb{C}[\mathcal{W}]$. To complete the proof use (14) together with Lemma 2.3 and the the obvious identity

$$
(k \cdot f)_{a}=k \cdot f_{a},
$$

$(k \in \operatorname{Sp}(n, \mathbb{F}), \quad f \in \mathbb{C}[H], \quad a \in \mathbb{F})$.

Two immediate but useful properties of Gelfand pairs are noted in the following lemma.

Lemma 2.8 Let $K_{1}$ and $K_{2}$ be a pair of subgroups of $S p(n, \mathbb{F})$ and suppose that $\left(K_{1}, H\right)$ a Gelfand pair.

(a) If $K_{1} \subset K_{2}$ then $\left(K_{2}, H\right)$ is a Gelfand pair.

(b) If $K_{1}, K_{2}$ are conjugate in $\operatorname{Sp}(n, \mathbb{F})$ then $\left(K_{2}, H\right)$ is a Gelfand pair.

\section{Gelfand pairs and the oscillator representation}

\subsection{Operator valued Fourier transform on $\mathbb{C}[\mathcal{W}]$}

For $f \in \mathbb{C}[\mathcal{W}]$ and $\lambda \in \mathbb{F}^{\times}$let $\pi_{\lambda}(f)$ denote the operator

$$
\pi_{\lambda}(f)=\sum_{\mathbf{z} \in \mathcal{W}} f(\mathbf{z}) \pi_{\lambda}(\mathbf{z})
$$

on $\mathbb{C}[\mathcal{W}]$. Here $\pi_{\lambda}(\mathbf{z})=\pi_{\lambda}(\mathbf{z}, 0)$ and $\pi_{\lambda}$ is the Schrödinger representation (8). The following standard result is easily verified.

Lemma $3.1 \pi_{\lambda}\left(f \natural_{\lambda} g\right)=\pi_{\lambda}(f) \pi_{\lambda}(g)$ for $f, g \in \mathbb{C}[\mathcal{W}]$ and $\lambda \in \mathbb{F}^{\times}$.

Lemma 3.2 The map $\pi_{\lambda}: \mathbb{C}[\mathcal{W}] \rightarrow \operatorname{End}\left(\mathbb{C}\left[\mathbb{F}^{n}\right]\right)$ is a vector space isomorphism for each $\lambda \in \mathbb{F}^{\times}$. In fact $q^{-3 n / 2} \pi_{\lambda}$ is a unitary isomorphism of $\mathbb{C}[\mathcal{W}]$ onto End $\left(\mathbb{C}\left[\mathbb{F}^{n}\right]\right)$ equipped with the Hilbert-Schmidt inner product

$$
\langle T, S\rangle_{H S}=\operatorname{tr}\left(T S^{*}\right)
$$


Proof. The set $\left\{q^{n / 2} \delta_{\mathbf{u}}: \mathbf{u} \in \mathbb{F}^{n}\right\}$ is an orthonormal basis for $\mathbb{C}\left[\mathbb{F}^{n}\right]$ with

$$
\pi_{\lambda}(\mathbf{x}, \mathbf{y}) \delta_{\mathbf{u}}=\psi_{\lambda}\left(\mathbf{u} \cdot \mathbf{y}-2^{-1} \mathbf{x} \cdot \mathbf{y}\right) \delta_{\mathbf{u}-\mathbf{x}} .
$$

So for $f, f^{\prime} \in \mathbb{C}[\mathcal{W}]$ we compute

$$
\begin{aligned}
\left\langle\pi_{\lambda}(f), \pi_{\lambda}\left(f^{\prime}\right)\right\rangle_{H S} & =\sum_{\mathbf{u} \in \mathbb{F}^{n}} q^{n}\left\langle\pi_{\lambda}(f) \delta_{\mathbf{u}}, \pi_{\lambda}\left(f^{\prime}\right) \delta_{\mathbf{u}}\right\rangle_{\mathbb{F}^{n}} \\
& =\sum_{\mathbf{u} \in \mathbb{F}^{n} ; \mathbf{z}, \mathbf{z}^{\prime} \in \mathcal{W}} f(\mathbf{z}), \overline{f^{\prime}\left(\mathbf{z}^{\prime}\right)} q^{n}\left\langle\pi_{\lambda}(\mathbf{z}) \delta_{\mathbf{u}}, \pi_{\lambda}\left(\mathbf{z}^{\prime}\right) \delta_{\mathbf{u}}\right\rangle_{\mathbb{F}^{n}} .
\end{aligned}
$$

A calculation using (8) shows

$$
\sum_{\mathbf{u} \in \mathbb{F}^{n}}\left\langle\pi_{\lambda}(\mathbf{z}) \delta_{\mathbf{u}}, \pi_{\lambda}\left(\mathbf{z}^{\prime}\right) \delta_{\mathbf{u}}\right\rangle_{\mathbb{F}^{n}}=\delta_{\mathbf{z}, \mathbf{z}^{\prime}}
$$

Thus

$$
\left\langle\pi_{\lambda}(f), \pi_{\lambda}\left(f^{\prime}\right)\right\rangle_{H S}=q^{n} \sum_{\mathbf{z} \in \mathcal{W}} f(\mathbf{z}) \overline{f^{\prime}(\mathbf{z})}=q^{3 n}\left\langle f, f^{\prime}\right\rangle_{\mathcal{W}}
$$

This shows $q^{-3 n / 2} \pi_{\lambda}: \mathbb{C}[\mathcal{W}] \rightarrow \operatorname{End}\left(\mathbb{C}\left[\mathbb{F}^{n}\right]\right)$ is unitary, hence injective. As the spaces $\mathbb{C}[\mathcal{W}]$ and $\operatorname{End}\left(\mathbb{C}\left[\mathbb{F}^{n}\right]\right)$ have equal dimension it follows that $\pi_{\lambda}$ is an isomorphism of $\mathbb{C}[\mathcal{W}]$ onto $\operatorname{End}\left(\mathbb{C}\left[\mathbb{F}^{n}\right]\right)$.

\subsection{Oscillator representation}

Recall that $\omega_{\lambda}: S p(n, \mathbb{F}) \rightarrow U\left(\mathbb{C}\left[\mathbb{F}^{n}\right]\right)$ denotes the oscillator representation, characterized by Equation 10 . Now for $k \in S p(n, \mathbb{F})$ let $\widetilde{\omega}_{\lambda}(k)$ be the operator on $\operatorname{End}\left(\mathbb{C}\left[\mathbb{F}^{n}\right]\right)$ defined as

$$
\widetilde{\omega}_{\lambda}(k) T=\omega_{\lambda}(k) T \omega_{\lambda}(k)^{-1}
$$

One checks that $\widetilde{\omega}_{\lambda}$ defines a unitary representation of $S p(n, \mathbb{F})$ on the Hermitian vector space $\left(\operatorname{End}\left(\mathbb{C}\left[\mathbb{F}^{n}\right],\langle\cdot, \cdot\rangle_{H S}\right)\right.$. Moreover for $k \in S p(n, \mathbb{F})$ and $f \in \mathbb{C}[\mathcal{W}]$ one has

$$
\pi_{\lambda}(k \cdot f)=\sum_{\mathbf{z} \in \mathcal{W}} f\left(k^{-1} \mathbf{z}\right) \pi_{\lambda}(\mathbf{z})=\sum_{\mathbf{w} \in \mathcal{W}} f(\mathbf{w}) \pi_{\lambda}(k \mathbf{w})=\widetilde{\omega}_{\lambda}(k) \pi_{\lambda}(f),
$$

since $\pi_{\lambda}(k \mathbf{w})=\omega_{\lambda}(k) \pi_{\lambda}(\mathbf{w}) \omega_{\lambda}(k)^{-1}$. So the isomorphism $\pi_{\lambda}: \mathbb{C}[\mathcal{W}] \rightarrow$ $\operatorname{End}\left(\mathbb{C}\left[\mathbb{F}^{n}\right]\right)$ intertwines the natural representation of $\operatorname{Sp}(n, \mathbb{F})$ on $\mathbb{C}[\mathcal{W}]$ with $\widetilde{\omega}_{\lambda}$.

Definition 3.3 Let $K$ be a subgroup of $S p(n, \mathbb{F})$. For $\lambda \in \mathbb{F}^{\times}$we define the commutant $\mathcal{C}_{\lambda, K}$ of $\widetilde{\omega}_{\lambda}(K)$ in $\operatorname{End}\left(\mathbb{C}\left[\mathbb{F}^{n}\right]\right)$ as

$\mathcal{C}_{\lambda, K}=\operatorname{End}\left(\mathbb{C}\left[\mathbb{F}^{n}\right]\right)^{\widetilde{\omega}_{\lambda}(K)}=\left\{T \in \operatorname{End}\left(\mathbb{C}\left[\mathbb{F}^{n}\right]\right): \omega_{\lambda}(k) T=T \omega_{\lambda}(k) \forall k \in K\right\}$.

Note that $\mathcal{C}_{\lambda, K}$ is a subalgebra of $\operatorname{End}\left(\mathbb{C}\left[\mathbb{F}^{n}\right]\right)$. 
Proposition $3.4 \pi_{\lambda}$ yields an algebra isomorphism of $\left(\mathbb{C}[\mathcal{W}]^{K}, \natural_{\lambda}\right)$ onto $\mathcal{C}_{\lambda, K}$

Proof. Taken together, Lemmas 3.1 and 3.2 show that $\pi_{\lambda}: \mathbb{C}[\mathcal{W}] \rightarrow \operatorname{End}\left(\mathbb{C}\left[\mathbb{F}^{n}\right]\right)$ is an algebra isomorphism of $\left(\mathbb{C}[\mathcal{W}], \mathfrak{\natural}_{\lambda}\right)$ onto $\operatorname{End}\left(\mathbb{C}\left[\mathbb{F}^{n}\right]\right)$. Equation 17 shows that $\pi_{\lambda}$ maps $\mathbb{C}[\mathcal{W}]^{K}$ onto $\mathcal{C}_{\lambda, K}$.

Proposition 3.5 Let $K$ be a subgroup of $\operatorname{Sp}(n, \mathbb{F})$ and $\lambda \in \mathbb{F}^{\times}$. Then $\left.\omega_{\lambda}\right|_{K}$ is multiplicity free if and only if $\mathcal{C}_{\lambda, K}$ is commutative.

Proof. Suppose that $\left.\omega_{\lambda}\right|_{K}$ is multiplicity free. So $\mathbb{C}\left[\mathbb{F}^{n}\right]$ has a canonical decomposition into pair-wise inequivalent $\omega_{\lambda}(K)$-irreducible subspaces:

$$
\mathbb{C}\left[\mathbb{F}^{n}\right]=P_{1} \oplus \cdots \oplus P_{m}
$$

say. Schur's Lemma shows that each operator $T \in \mathcal{C}_{\lambda, K}$ must preserve the $P_{j}$ 's and act by a scalar on each. Any two such operators commute with one another.

Next suppose that $\left.\omega_{\lambda}\right|_{K}$ is not multiplicity free. Hence $\mathbb{C}\left[\mathbb{F}^{n}\right]$ has a decomposition of the sort

$$
\mathbb{C}\left[\mathbb{F}^{n}\right]=W_{1} \oplus W_{2} \oplus V
$$

where $W_{1}, W_{2}, V$ are $\omega_{\lambda}(K)$-invariant and $W_{1}, W_{2}$ are $\omega_{\lambda}(K)$-irreducible and equivalent. Thus $\mathcal{C}_{\lambda, K}$ contains a copy of $G L(2, \mathbb{F})$, and it fails to be commutative.

Definition 3.6 We say that a subgroup $K$ of $S p(n, \mathbb{F})$ is $\omega$-multiplicity free if the restriction $\left.\omega_{\lambda}\right|_{K}$ of the oscillator representation to $K$ is multiplicity free for all $\lambda \in \mathbb{F}^{\times}$.

Together Propositions 2.7, 3.4 and 3.5 imply the following.

Theorem 3.7 Let $K$ be a subgroup of $S p(n, \mathbb{F})$. Then $(K, H)$ is a Gelfand pair if and only if $K$ is $\omega$-multiplicity free.

When applying Definition 3.6 it suffices to check that $\left.\omega_{\lambda}\right|_{K}$ is multiplicity free for at most two values of $\lambda$.

Proposition 3.8 A subgroup $K$ of $S p(n, \mathbb{F})$ is $\omega$-multiplicity free if and only if $\left.\omega_{1}\right|_{K}$ and $\left.\omega_{\varepsilon}\right|_{K}$ are multiplicity free for any fixed choice of $\varepsilon \in \mathbb{F}^{\times}$which is not a square. Moreover when $q \equiv 3 \bmod 4$ it suffices that $\left.\omega_{1}\right|_{K}$ be multiplicity free.

Proof. Proposition 1.2 implies that each oscillator representation $\omega_{\lambda}$ is unitarily equivalent to one of $\omega_{1}$ or $\omega_{\varepsilon}$. If -1 is not a square in $\mathbb{F}$, equivalently when $q \equiv 3 \bmod 4$, we can take $\varepsilon=-1$. But $\omega_{-1}$ is contragredient to $\omega_{1}$ by (11). So when $\left.\omega_{1}\right|_{K}$ is multiplicity free so is $\left.\omega_{-1}\right|_{K}$.

Remark 3.9 We do not know of an example where $\left.\omega_{1}\right|_{K}$ is multiplicity free but $K$ fails to be $\omega$-multiplicity free. 


\section{Counting and convolving $K$-orbits in $\mathcal{W}$}

Let $\rho$ denote the natural (unitary) representation of $S p(n, \mathbb{F})$ on $\mathbb{C}[\mathcal{W}]$ :

$$
\rho(k) f(\mathbf{z})=k \cdot f(\mathbf{z})=f\left(k^{-1} \mathbf{z}\right),
$$

and $\widetilde{\omega}_{\lambda}: S p(n, \mathbb{F}) \rightarrow U\left(\operatorname{End}\left(\mathbb{C}\left[\mathbb{F}^{n}\right]\right)\right.$ be as in (16). We have seen that $\rho$ and $\widetilde{\omega}_{\lambda}$ are unitarily equivalent via $q^{-3 n / 2} \pi_{\lambda}$, a multiple of the operator valued Fourier transform. There is another viewpoint on this equivalence. Consider the standard isomorphism

$$
\Phi: \mathbb{C}\left[\mathbb{F}^{n}\right] \otimes \mathbb{C}\left[\mathbb{F}^{n}\right]^{*} \rightarrow \operatorname{End}\left(\mathbb{C}\left[F^{n}\right]\right), \quad \Phi(f \otimes \varphi)(g)=\varphi(g) f .
$$

One checks easily that

- $\Phi$ is unitary. (As before $\operatorname{End}\left(\mathbb{C}\left[F^{n}\right]\right)$ carries the Hilbert-Schmidt inner product and we give $\mathbb{C}\left[\mathbb{F}^{n}\right] \otimes \mathbb{C}\left[\mathbb{F}^{n}\right]^{*}$ the tensor product of $\langle\cdot, \cdot\rangle_{\mathbb{F}^{n}}$ with its dual inner product.)

- $\Phi\left(\omega_{\lambda}(k) f \otimes \omega_{\lambda}^{*}(k) \varphi\right)=\widetilde{\omega}_{\lambda}(k) \Phi(f \otimes \varphi)$.

So $\Phi$ establishes a unitary equivalence

$$
\omega_{\lambda} \otimes \omega_{\lambda}^{*} \simeq \widetilde{\omega}_{\lambda}
$$

and the composite $q^{3 n / 2} \pi_{\lambda}^{-1} \circ \Phi$ yields

$$
\omega_{\lambda} \otimes \omega_{\lambda}^{*} \simeq \rho .
$$

This basic fact plays a central role in [How].

\subsection{Counting orbits}

Now let $K$ be a subgroup of $S p(n, \mathbb{F})$ and for $\lambda \in \mathbb{F}^{\times}$decompose $\left.\omega_{\lambda}\right|_{K}$ :

$$
\left.\omega_{\lambda}\right|_{K} \simeq \sum_{\sigma \in \widehat{K}} m_{\sigma, \lambda} \sigma, \quad m_{\sigma, \lambda}=\operatorname{mult}\left(\sigma,\left.\omega_{\lambda}\right|_{K}\right) .
$$

Writing $d_{\sigma}=\operatorname{dim}(\sigma)$ one has

$$
\sum_{\sigma \in \widehat{K}} m_{\sigma, \lambda} d_{\sigma}=\operatorname{dim}\left(\mathbb{C}\left[\mathbb{F}^{n}\right]\right)=q^{n} .
$$

Also, applying (18):

$$
\left.\left.\rho\right|_{K} \simeq\left(\omega_{\lambda} \otimes \omega_{\lambda}^{*}\right)\right|_{K} \simeq \sum_{\sigma, \sigma^{\prime} \in \widehat{K}} m_{\sigma, \lambda} m_{\sigma^{\prime}, \lambda} \sigma \otimes\left(\sigma^{\prime}\right)^{*} .
$$

We know that $\sigma \otimes(\sigma)^{*}$ has a one-dimensional space of $K$-fixed vectors and that $\left(\sigma \otimes\left(\sigma^{\prime}\right)^{*}\right)^{K}=0$ when $\sigma^{\prime} \not 千 \sigma$. So 


$$
\operatorname{dim}\left(\mathbb{C}[\mathcal{W}]^{K}\right)=\sum_{\sigma \in \widehat{K}} m_{\sigma, \lambda}^{2}
$$

But $\operatorname{dim}\left(\mathbb{C}[\mathcal{W}]^{K}\right)=|\mathcal{W} / K|$, the number of $K$-orbits in $\mathcal{W}$. (Indeed, if $K \mathbf{z}_{1}, \ldots, K \mathbf{z}_{r}$ are the distinct $K$-orbits in $\mathcal{W}$ then the characteristic functions $\left\{\delta_{K \mathbf{z}_{1}}, \ldots, \delta_{K \mathbf{z}_{r}}\right\}$ form a basis for $\mathbb{C}[\mathcal{W}]^{K}$.) So now

$$
\sum_{\sigma \in \widehat{K}} m_{\sigma, \lambda}^{2}=|\mathcal{W} / K|
$$

Proposition 4.1 If $K$ is $\omega$-multiplicity free (equivalently $(K, H)$ is a Gelfand pair) then we must have

- $|\mathcal{W} / K| \leq q^{n}$ and

- $|K| \geq q^{n}+1$.

Proof. Suppose that $|\mathcal{W} / K|>q^{n}$. Using Equations 19 and 20 one obtains

$$
\sum_{\sigma \in \widehat{K}} m_{\sigma, \lambda}^{2}>\sum_{\sigma \in \widehat{K}} m_{\sigma, \lambda} d_{\sigma} \geq \sum_{\sigma \in \widehat{K}} m_{\sigma, \lambda} .
$$

So we must have $m_{\sigma, \lambda} \geq 2$ for some $\sigma \in \widehat{K}$. Hence $K$ fails to be $\omega$-multiplicity free unless $|\mathcal{W} / K| \leq q^{n}$.

Next observe that

$$
|\mathcal{W} / K| \geq 1+\frac{q^{2 n}-1}{|K|}
$$

since $\{0\}$ is a $K$-orbit in $\mathcal{W}$ and $\mathcal{W} \backslash\{0\}$ contains at least $\frac{q^{2 n}-1}{|K|} K$-orbits. So in view of the inequality $|\mathcal{W} / K| \leq q^{n}$ we must have

$$
1+\frac{q^{2 n}-1}{|K|} \leq q^{n} \Longrightarrow \frac{q^{2 n}-1}{q^{n}-1} \leq|K| \Longrightarrow q^{n}+1 \leq|K|,
$$

as claimed.

Proposition 4.2 Let $K$ be an abelian subgroup of $S p(n, \mathbb{F})$. Then $|\mathcal{W} / K| \geq$ $q^{n}$ and $K$ is $\omega$-multiplicity free if and only if $|\mathcal{W} / K|=q^{n}$. Moreover, this is possible only when $|K| \geq q^{n}+1$.

Proof. When $K$ is abelian we have $d_{\sigma}=1$ for all $\sigma \in \widehat{K}$. So Equations 19 and 20 become

$$
\sum_{\sigma \in \widehat{K}} m_{\sigma, \lambda}=q^{n}, \quad \sum_{\sigma \in \widehat{K}} m_{\sigma, \lambda}^{2}=|\mathcal{W} / K| .
$$

As $\sum_{\sigma \in \widehat{K}} m_{\sigma, \lambda}^{2} \geq \sum_{\sigma \in \widehat{K}} m_{\sigma, \lambda}$ we conclude that $|\mathcal{W} / K| \geq q^{n}$ must hold. Also $K$ is $\omega$-multiplicity free if and only if $\sum_{\sigma \in \widehat{K}} m_{\sigma, \lambda}^{2}=\sum_{\sigma \in \widehat{K}} m_{\sigma, \lambda}$. Equivalently, $|\mathcal{W} / K|=q^{n}$ must hold. Proposition 4.1 shows, moreover, that this implies $|K| \geq q^{n}+1$. 


\subsection{Convolving orbits}

The characteristic functions

$$
\left\{\delta_{K \mathbf{z}}: K \mathbf{z} \in \mathcal{W} / K\right\}
$$

for the $K$-orbits in $\mathcal{W}$ yield an (orthogonal) basis for $\mathbb{C}[\mathcal{W}]^{K}$. We compute

$$
\begin{aligned}
\delta_{K \mathbf{z}} \natural_{\lambda} \delta_{K \mathbf{z}^{\prime}}(\mathbf{w}) & =\sum_{\mathbf{v} \in \mathcal{W}} \delta_{K \mathbf{z}}(\mathbf{w}-\mathbf{v}) \delta_{K \mathbf{z}^{\prime}}(\mathbf{v}) \psi_{\lambda}\left(2^{-1}[\mathbf{w}, \mathbf{v}]\right) \\
& =\sum_{\mathbf{v} \in(\mathbf{w}-K \mathbf{z}) \cap K \mathbf{z}^{\prime}} \psi_{\lambda}\left(2^{-1}[\mathbf{w}, \mathbf{v}]\right) .
\end{aligned}
$$

In view of Proposition 2.7, $(K, H)$ will be a Gelfand pair if and only if $\delta_{K \mathbf{z}} \natural_{\lambda} \delta_{K \mathbf{z}^{\prime}}(\mathbf{w})=\delta_{K \mathbf{z}^{\prime}} \mathfrak{\natural}_{\lambda} \delta_{K \mathbf{z}}(\mathbf{w})$ for all $\mathbf{z}, \mathbf{z}^{\prime}, \mathbf{w} \in \mathcal{W}$ and all $\lambda \in \mathbb{F}^{\times}$. It is enough to consider $\mathbf{z} \neq 0 \neq \mathbf{z}^{\prime}$ since $\delta_{K 0}=\delta_{0}$ is a two-sided identity in $\left(\mathbb{C}[\mathcal{W}], \bigsqcup_{\lambda}\right)$. Moreover we can take $\mathbf{w} \neq 0$ because, in any case, $f \natural_{\lambda} g(0)=f \natural_{\lambda} g(0)$ for functions $f, g \in \mathbb{C}[\mathcal{W}]$. This discussion yields the following.

Lemma $4.3(K, H)$ is a Gelfand pair if and only if

$$
\sum_{\mathbf{v} \in(\mathbf{w}-K \mathbf{z}) \cap K \mathbf{z}^{\prime}} \psi_{\lambda}([\mathbf{w}, \mathbf{v}])=\sum_{\mathbf{v} \in\left(\mathbf{w}-K \mathbf{z}^{\prime}\right) \cap K \mathbf{z}} \psi_{\lambda}([\mathbf{w}, \mathbf{v}])
$$

for all $\mathbf{z}, \mathbf{z}^{\prime}, \mathbf{w} \in \mathcal{W} \backslash\{0\}$ and $\lambda \in \mathbb{F}^{\times}$.

Remark 4.4 By Proposition 3.8 it suffices to check the condition in Lemma 4.3 for at most two values of the parameter $\lambda$.

Lemma 4.5 Suppose that there are $K$-invariant subspaces $\mathcal{X}$ and $\mathcal{Y}$ in $\mathcal{W}$ with

$$
\mathcal{X} \cap \mathcal{Y}=0, \quad[\mathcal{X}, \mathcal{Y}] \neq 0 .
$$

Then $(K, H)$ is not a Gelfand pair.

Proof. Choose points $\mathbf{z} \in \mathcal{X}$ and $\mathbf{z}^{\prime} \in \mathcal{Y}$ with $\left[\mathbf{z}, \mathbf{z}^{\prime}\right]=1$ and let $\mathbf{w}=\mathbf{z}+\mathbf{z}^{\prime}$. We have

$$
(\mathbf{w}-K \mathbf{z}) \cap K \mathbf{z}^{\prime}=\left\{\mathbf{z}^{\prime}\right\} .
$$

Indeed, suppose that $\mathbf{v} \in(\mathbf{w}-K \mathbf{z}) \cap K \mathbf{z}^{\prime}$. Thus for some $k, k^{\prime} \in K$,

$$
\mathbf{w}-k \mathbf{z}=\mathbf{v}=k^{\prime} \mathbf{z}^{\prime} \quad \text { and hence } \quad \mathbf{z}-k \mathbf{z}=k^{\prime} \mathbf{z}^{\prime}-\mathbf{z}^{\prime} .
$$

But $\mathbf{z}-k \mathbf{z} \in \mathcal{X}$ and $k^{\prime} \mathbf{z}^{\prime}-\mathbf{z}^{\prime} \in \mathcal{Y}$. So $\mathbf{v}=k^{\prime} \mathbf{z}^{\prime}=\mathbf{z}^{\prime}$ since $\mathcal{X} \cap \mathcal{Y}=0$.

Thus for these choices of $\mathbf{z}, \mathbf{z}^{\prime}$ and $\mathbf{w}$ we have

$$
\sum_{\mathbf{v} \in(\mathbf{w}-K \mathbf{z}) \cap K \mathbf{z}^{\prime}} \psi_{1}([\mathbf{w}, \mathbf{v}])=\psi_{1}\left(\left[\mathbf{z}+\mathbf{z}^{\prime}, \mathbf{z}^{\prime}\right]\right)=\psi_{1}(1)=e^{2 \pi i / p} .
$$


Likewise

$$
\sum_{\mathbf{v} \in\left(\mathbf{w}-K \mathbf{z}^{\prime}\right) \cap K \mathbf{z}} \psi_{1}([\mathbf{w}, \mathbf{v}])=\psi_{1}\left(\left[\mathbf{z}+\mathbf{z}^{\prime}, \mathbf{z}\right]\right)=\psi_{1}(-1)=e^{-2 \pi i / p} .
$$

As $p$ is odd, these values are necessarily different. So $(K, H)$ fails to be a Gelfand pair in view of Lemma 4.3.

Corollary 4.6 Let $\left(K, H_{\mathcal{W}}\right)$ be a Gelfand pair where $K$ acts reductively but non-irreducibly on $\mathcal{W}$. So $\mathcal{W}$ decomposes as a direct sum of $K$-invariant subspaces,

$$
\mathcal{W}=\mathcal{W}_{1} \oplus \cdots \oplus \mathcal{W}_{\ell}
$$

say. Let $K_{j} \subset G L\left(\mathcal{W}_{j}\right)$ denote the restriction of $K$ to $\mathcal{W}_{j}$. Then:

(a) $\left[\mathcal{W}_{i}, \mathcal{W}_{j}\right]=0$ for all $i \neq j$.

(b) Each $\mathcal{W}_{j}$ is a symplectic subspace of $\mathcal{W}$ and $K_{j} \subset S p\left(\mathcal{W}_{j}\right)$.

(c) Each $\left(K_{j}, H_{\mathcal{W}_{j}}\right)$ is a Gelfand pair.

Corollary 4.7 Suppose we are given symplectic vector spaces $\left(\mathcal{W}_{j},[\cdot, \cdot]_{j}\right)$ and subgroups $K_{j} \subset S p\left(\mathcal{W}_{j}\right)$ for $j=1, \ldots \ell$. We form the symplectic direct sum

$$
W=\mathcal{W}_{1} \oplus \cdots \oplus \mathcal{W}_{\ell}, \quad\left[\left(\mathbf{w}_{1}, \ldots, \mathbf{w}_{\ell}\right),\left(\mathbf{w}_{1}^{\prime}, \ldots, \mathbf{w}_{\ell}^{\prime}\right)\right]=\sum_{j=1}^{\ell}\left[\mathbf{w}_{j}, \mathbf{w}_{j}^{\prime}\right]_{j}
$$

and let $K \subset S p(\mathcal{W})$ denote the product

$$
K=K_{1} \times \cdots \times K_{\ell}
$$

Then $\left(K, H_{\mathcal{W}}\right)$ is a Gelfand pair if and only if $\left(K_{j}, H_{\mathcal{W}_{j}}\right)$ is a Gelfand pair for $j=1, \ldots, \ell$.

Recall that a subspace $\mathcal{X}$ of the symplectic vector space $\mathcal{W}$ is said to be isotropic if $[\mathcal{X}, \mathcal{X}]=0$.

Corollary 4.8 Suppose that $K$ acts reductively on $\mathcal{W}$ and that the action preserves a non-zero isotropic subspace $\mathcal{X}$. Then $(K, H)$ is not a Gelfand pair.

Proof. As $K$ acts reductively on $\mathcal{W}$ we have $\mathcal{W}=\mathcal{X} \oplus \mathcal{Y}$ for some $K$-invariant subspace $\mathcal{Y}$. As $\mathcal{X}$ is isotropic we necessarily have $[\mathcal{X}, \mathcal{Y}] \neq 0$.

\section{Examples}

\subsection{Symplectic groups}

A trivial application of Lemma 4.3 shows that $\left(S p(n, \mathbb{F}), H_{n}(\mathbb{F})\right)$ is a Gelfand pair. Indeed, for $K=S p(n, \mathbb{F})$ we have only one non-zero $K$-orbit. That is 
$K \mathbf{z}=\mathcal{W} \backslash\{0\}$ for all $\mathbf{z} \neq 0$ in $\mathcal{W}$. Equation 20 now shows that the oscillator representation $\omega_{\lambda}$ must decompose into exactly two inequivalent irreducible constituents. This fact is well known. (See [How], [Neu02].) The $\omega_{\lambda}(S p(n, \mathbb{F}))-$ irreducible subspaces of $\mathbb{C}\left[\mathbb{F}^{n}\right]$ can be identified as follows.

The matrix $-I$ belongs to the center of $S p(n, \mathbb{F})$ and the first formula from Theorem 1.3 shows that $\omega_{\lambda}(-I)=\operatorname{sgn}\left((-1)^{n}\right) T$ where

$$
T f(\mathbf{u})=f(-\mathbf{u}) \text {. }
$$

So the eigenspaces for $T$ must be $\omega_{\lambda}(S p(n, \mathbb{F}))$-invariant. These are the spaces of even and odd functions. As $\omega_{\lambda}$ has exactly two irreducible components these spaces are necessarily irreducible.

To obtain more interesting examples we must consider smaller subgroups of $\operatorname{Sp}(n, \mathbb{F})$.

\subsection{General linear groups}

The group $G L(n, \mathbb{F})$ embeds diagonally in $S p(n, \mathbb{F})$ via $\left\{A_{\text {diag }}: A \in\right.$ $G L(n, \mathbb{F})\}$. (See (12).) The action of $G L(n, \mathbb{F})$ on $\mathcal{W}=\mathbb{F}^{n} \times \mathbb{F}^{n}$ preserves the isotropic subspaces

$$
\mathcal{X}=\mathbb{F}^{n} \times\{0\}, \quad \mathcal{Y}=\{0\} \times \mathbb{F}^{n} .
$$

So $\left(G L(n, \mathbb{F}), H_{n}(\mathbb{F})\right)$ is not a Gelfand pair, in view of Corollary 4.8. More generally, if $K \subset S p(n, \mathbb{F})$ is conjugate in $S p(n, \mathbb{F})$ to a subgroup of $G L(n, \mathbb{F})$ then $(K, H)$ is not a Gelfand pair.

For $n \geq 2$ there are $q+3$ distinct $G L(n, \mathbb{F})$-orbits in $\mathcal{W}$, namely

$$
\begin{gathered}
\{(0,0)\}, \quad\left(\mathbb{F}^{n} \backslash\{0\}\right) \times\{0\}, \quad\{0\} \times\left(\mathbb{F}^{n} \backslash\{0\}\right), \\
\{(\mathbf{x}, \mathbf{y}): \mathbf{x} \neq 0 \neq \mathbf{y}, \mathbf{x} \cdot \mathbf{y}=0\} \quad \text { and } \quad\{(\mathbf{x}, \mathbf{y}): \mathbf{x} \cdot \mathbf{y}=a\}
\end{gathered}
$$

for each $a \in \mathbb{F}^{\times}$. So here $|\mathcal{W} / G L(n, \mathbb{F})|<q^{n}$, but $\left(G L(n, \mathbb{F}), H_{n}(\mathbb{F})\right)$ is not a Gelfand pair. This shows that, for $K$ non-abelian, there can be no converse for Proposition 4.1.

\subsection{Borel subgroups}

A subgroup of $S p(n, \mathbb{F})$ conjugate to

$$
B=\left\{\left[\begin{array}{c|c}
A \mid 0 \\
\hline C \mid\left(A^{t}\right)^{-1}
\end{array}\right]: A \in G L(n, \mathbb{F}) \text { lower triangular, } A^{t} C \text { symmetric }\right\}
$$

is called a Borel subgroup.

Proposition 5.1 If $K$ is a subgroup of $\operatorname{Sp}(n, \mathbb{F})$ that contains a Borel subgroup then $(K, H)$ is a Gelfand pair. 
Proof. It suffices to show that $(B, H)$ is a Gelfand pair. This can be done using Proposition 2.6. There are exactly $2 n$ non-zero $B$-orbits in $\mathcal{W}=\mathbb{F}^{n} \times \mathbb{F}^{n}$ :

$$
\mathcal{W} \backslash\{0\}=\biguplus_{j=1}^{n} B \mathbf{u}_{j} \uplus \biguplus_{j=1}^{n} B \mathbf{v}_{j} \quad \text { where } \quad \mathbf{u}_{j}=\left(\mathbf{e}_{j}, 0\right), \mathbf{v}_{j}=\left(0, \mathbf{e}_{j}\right)
$$

and $\left\{\mathbf{e}_{j}\right\}$ is the standard basis for $\mathbb{F}^{n}$.

Suppose that $\mathbf{z} \in B \mathbf{u}_{i}$ and $\mathbf{w} \in B \mathbf{u}_{j}$. One has $\mathbf{z} \pm \mathbf{w} \in B \mathbf{u}_{\min (i, j)}$, and hence $\mathbf{z}+\mathbf{w}=k(\mathbf{z}-\mathbf{w})$ for some $k \in B$. Let $k^{\prime}=-k$ and note that $k^{\prime} \in B$, since $-I \in B$. Now

$$
\begin{aligned}
(\mathbf{z}, s)(\mathbf{w}, t) & =\left(\mathbf{z}+\mathbf{w}, s+t+2^{-1}[\mathbf{z}, \mathbf{w}]\right) \\
& =\left(k \mathbf{z}-k \mathbf{w}, s+t+2^{-1}[k \mathbf{z}, k \mathbf{w}]\right) \\
& =\left(k^{\prime} \mathbf{w}+k \mathbf{z}, t+s+2^{-1}\left[k^{\prime} \mathbf{w}, k \mathbf{z}\right]\right) \\
& =\left(k^{\prime} \mathbf{w}, t\right)(k \mathbf{z}, s) .
\end{aligned}
$$

So $(\mathbf{z}, s)(\mathbf{w}, t) \in(B(\mathbf{w}, t))(B(\mathbf{z}, s))$. Similar calculations apply for other combinations of orbits.

One can also give an explicit description of the algebra $\left(\mathbb{C}[\mathcal{W}]^{B}, \natural_{\lambda}\right)$ by determining the twisted convolution of pairs of characteristic functions for $B$-orbits in $\mathcal{W}$. We adopt the notation

$$
E_{\lambda}(\mathbf{z})=\sum_{\mathbf{w} \in B \mathbf{z}} \psi_{\lambda}\left(2^{-1}[\mathbf{z}, \mathbf{w}]\right) \quad\left(\mathbf{z} \in \mathcal{W}, \lambda \in \mathbb{F}^{\times}\right) .
$$

Brute force calculation yields the following.

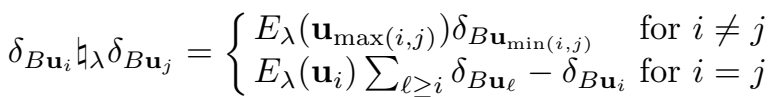

$$
\begin{aligned}
& \delta_{B \mathbf{v}_{i}} \natural_{\lambda} \delta_{B \mathbf{v}_{j}}= \begin{cases}E_{\lambda}\left(\mathbf{v}_{\min (i, j)}\right) \delta_{B \mathbf{v}_{\max (i, j)}} & \text { for } i \neq j \\
E_{\lambda}\left(\mathbf{v}_{i}\right) \sum_{\ell \leq i} \delta_{B \mathbf{v}_{\ell}}-\delta_{B \mathbf{v}_{i}} & \text { for } i=j\end{cases} \\
& \delta_{B \mathbf{u}_{j}} \natural_{\lambda} \delta_{B \mathbf{v}_{i}}=\delta_{B \mathbf{v}_{i}} \natural_{\lambda} \delta_{B \mathbf{u}_{j}}=E_{\lambda}\left(\mathbf{v}_{i}\right) \delta_{B \mathbf{u}_{j}}
\end{aligned}
$$

These formulas show, in particular, that $\left(\mathbb{C}[\mathcal{W}]^{B}, \natural_{\lambda}\right)$ is commutative, as guaranteed by Proposition 5.1.

\subsection{Unitary groups}

Let $\widetilde{\mathbb{F}}$ denote a quadratic extension of the field $\mathbb{F}=\mathbb{F}_{q}$. Up to isomorphism $\widetilde{\mathbb{F}}$ is a copy of $\mathbb{F}_{q^{2}}$. More concretely we choose any non-square

$$
\varepsilon \in \mathbb{F}^{\times} \backslash\left(\mathbb{F}^{\times}\right)^{2}
$$

in $\mathbb{F}$ and take 


$$
\widetilde{\mathbb{F}}=\mathbb{F}(\sqrt{\varepsilon}) .
$$

The Galois involution $\widetilde{\mathbb{F}} \rightarrow \widetilde{\mathbb{F}}$ will be written as $z \mapsto \bar{z}$. One has $\bar{z}=z^{q}$ and

$$
\overline{a+b \sqrt{\varepsilon}}=a-b \sqrt{\varepsilon} \quad(a, b \in \mathbb{F}) .
$$

Let $(\widetilde{\mathcal{W}},\langle\cdot, \cdot\rangle)$ be a (finite dimensional) Hermitian vector space over $\widetilde{\mathbb{F}}$. That is $\langle\cdot, \cdot\rangle: \widetilde{\mathcal{W}} \times \widetilde{\mathcal{W}} \rightarrow \widetilde{\mathbb{F}}$ is

- $\mathbb{F}$-bilinear and non-degenerate with

- $\left\langle\lambda \mathbf{z}, \mathbf{z}^{\prime}\right\rangle=\lambda\left\langle\mathbf{z}, \mathbf{z}^{\prime}\right\rangle$ and $\overline{\left\langle\mathbf{z}, \mathbf{z}^{\prime}\right\rangle}=\left\langle\mathbf{z}^{\prime}, \mathbf{z}\right\rangle \quad\left(\mathbf{z}, \mathbf{z}^{\prime} \in \widetilde{\mathcal{W}}, \lambda \in \widetilde{\mathbb{F}}\right)$.

The unitary group $U(\widetilde{\mathcal{W}})$ is the set of $\widetilde{\mathbb{F}}$-linear operators preserving $\langle\cdot, \cdot\rangle$.

Let $\mathcal{W}$ denote the underlying $\mathbb{F}$-vector space for $\widetilde{\mathcal{W}}$. One obtains a symplectic form on $\mathcal{W}$ via

$$
\left[\mathbf{z}, \mathbf{z}^{\prime}\right]=\frac{1}{2 \sqrt{\varepsilon}}\left(\overline{\left\langle\mathbf{z}, \mathbf{z}^{\prime}\right\rangle}-\left\langle\mathbf{z}, \mathbf{z}^{\prime}\right\rangle\right)=\frac{1}{2 \sqrt{\varepsilon}}\left(\left\langle\mathbf{z}^{\prime}, \mathbf{z}\right\rangle-\left\langle\mathbf{z}, \mathbf{z}^{\prime}\right\rangle\right) .
$$

(Writing $\left\langle\mathbf{z}, \mathbf{z}^{\prime}\right\rangle \in \mathbb{F}(\sqrt{\varepsilon})$ as $\left\langle\mathbf{z}, \mathbf{z}^{\prime}\right\rangle=\left\langle\mathbf{z}, \mathbf{z}^{\prime}\right\rangle_{r}+\left\langle\mathbf{z}, \mathbf{z}^{\prime}\right\rangle_{i} \sqrt{\varepsilon}$, one has $\left[\mathbf{z}, \mathbf{z}^{\prime}\right]=$ $-\left\langle\mathbf{z}, \mathbf{z}^{\prime}\right\rangle_{i}$.) Clearly $U(\widetilde{\mathcal{W}})$ is a subgroup of $S p(\mathcal{W})$, the symplectic group for $(\mathcal{W},[\cdot, \cdot])$. We will prove that:

Proposition $5.2\left(U(\widetilde{\mathcal{W}}), H_{\mathcal{W}}\right)$ is a Gelfand pair.

It is well known that a given finite dimensional $\widetilde{\mathbb{F}}$-vector space admits exactly one hermitian inner product, up to equivalence. ${ }^{1}$ In fact one can find an orthonormal basis

$$
\mathcal{B}=\left\{e_{1}, \ldots, e_{n}\right\}
$$

for $(\widetilde{\mathcal{W}},\langle\cdot, \cdot\rangle)$ with $\left\langle e_{i}, e_{j}\right\rangle=\delta_{i, j}$. Using $\mathcal{B}$ to identify $\widetilde{\mathcal{W}}$ with $\widetilde{\mathbb{F}}^{n}$ we have the usual formula

$$
\left\langle\mathbf{z}, \mathbf{z}^{\prime}\right\rangle=z_{1} \overline{z_{1}^{\prime}}+\cdots+z_{n} \overline{z_{n}^{\prime}} .
$$

Let

$\widetilde{\mathcal{W}}_{j}=\widetilde{\mathbb{F}} e_{j}, \quad \mathcal{W}_{j}$ denote $\widetilde{W}_{j}$ viewed as an $\mathbb{F}$-vector space, $\quad f_{j}=\sqrt{\varepsilon} e_{j}$.

Now

- $\left\{e_{j}, f_{j}\right\}$ is a basis for $\mathcal{W}_{j}$,

- $\left\{e_{1}, \ldots, e_{n}, f_{1}, \ldots, f_{n}\right\}$ is a symplectic basis for $\mathcal{W}$ (i.e. $\left[e_{i}, e_{j}\right]=0=$ $\left.\left[f_{i}, f_{j}\right],\left[e_{i}, f_{j}\right]=\delta_{i, j}\right)$, and

- $\mathcal{W}=\mathcal{W}_{1} \oplus \cdots \oplus \mathcal{W}_{n}$ is a symplectic direct sum.

${ }^{1}$ In contrast, the complex vector space $\mathbb{C}^{n}$ admits $\lfloor(n+2) / 2\rfloor$ inequivalent Hermitian inner products. These yield distinct unitary groups $U(r, s)$ with $r+s=n$. The analogs for these Hermitian inner products in the finite fields context are, however, mutually equivalent. 
The restriction of $\langle\cdot, \cdot\rangle$ to $\widetilde{\mathcal{W}}_{j}$ is a hermitian inner product on $\widetilde{\mathcal{W}}_{j}$. We consider the subgroups $U\left(\widetilde{\mathcal{W}}_{i}\right) \subset S p\left(\mathcal{W}_{j}\right)$ and their direct product

$$
U\left(\widetilde{\mathcal{W}}_{1}\right) \times \cdots \times U\left(\widetilde{\mathcal{W}}_{n}\right) \subset U(\widetilde{\mathcal{W}}) \subset S p(\mathcal{W}),
$$

the subgroup of $U(\widetilde{\mathcal{W}})$ preserving the decomposition $\mathcal{W}=\mathcal{W}_{1} \oplus \cdots \oplus \mathcal{W}_{n}$.

The following result evidentally implies Proposition 5.2.

Proposition $5.3\left(U\left(\widetilde{\mathcal{W}}_{1}\right) \times \cdots \times U\left(\widetilde{\mathcal{W}}_{n}\right), H_{\mathcal{W}}\right)$ is a Gelfand pair.

Proof. In view of Corollary 4.7 it suffices to show that each $\left(U\left(\widetilde{\mathcal{W}}_{j}\right), H_{\mathcal{W}_{j}}\right)$ is a Gelfand pair. This amounts to showing that $\left(U(\widetilde{\mathbb{F}}), H_{1}(\mathbb{F})\right)$ is a Gelfand pair, where $\widetilde{\mathbb{F}}$ carries the Hermitian inner product

$$
\left\langle z, z^{\prime}\right\rangle=z \overline{z^{\prime}} .
$$

Now

$$
U(\widetilde{\mathbb{F}})=\left\{\lambda \in \widetilde{\mathbb{F}}^{\times}: \lambda \bar{\lambda}=1\right\},
$$

is the kernel of the norm mapping

$$
N: \widetilde{\mathbb{F}} \rightarrow \mathbb{F}, \quad N(\lambda)=\lambda \bar{\lambda}
$$

restricted to the multiplicative group $\widetilde{\mathbb{F}}^{\times}$for the field $\widetilde{\mathbb{F}}$. So $U(\widetilde{\mathbb{F}})$ is, in particular, abelian. Moreover a pair of points $z, z^{\prime} \in \widetilde{\mathbb{F}}$ belong to a common $U(\widetilde{\mathbb{F}})$-orbit in $\widetilde{\mathbb{F}}$ if and only if $N(z)=N\left(z^{\prime}\right)$. So

$$
|\widetilde{\mathbb{F}} / U(\widetilde{\mathbb{F}})|=|N(\widetilde{\mathbb{F}})|=|\mathbb{F}|=q,
$$

as it is well known that $N$ is surjective. Proposition 4.2 now implies that $\left(U(\widetilde{\mathbb{F}}), H_{1}(\mathbb{F})\right)$ is a Gelfand pair as desired.

Remark 5.4 As $U(\widetilde{\mathbb{F}})$ is the kernel of the epimorphism $N: \widetilde{\mathbb{F}}^{\times} \rightarrow \mathbb{F}^{\times}$, it is cyclic of order $q+1$.

Our final result asserts that the Gelfand pair in Proposition 5.3 is minimal. The analogous theorem for real Heisenberg groups $H_{n}(\mathbb{R})$ is due to Leptin [Lep85].

Proposition $5.5\left(K, H_{\mathcal{W}}\right)$ fails to be a Gelfand pair for all proper subgroups $K$ of the torus $U\left(\widetilde{\mathcal{W}}_{1}\right) \times \cdots \times U\left(\widetilde{\mathcal{W}}_{n}\right)$.

Proof. We can take $\widetilde{\mathcal{W}}_{j}=\widetilde{\mathbb{F}}$ and $\widetilde{\mathcal{W}}=\widetilde{\mathbb{F}}^{n}$ with the usual hermitian inner product. Now the torus

$$
T=U(\widetilde{\mathbb{F}}) \times \cdots \times U(\widetilde{\mathbb{F}})
$$

coincides with a $T$-orbit in $\widetilde{\mathcal{W}}$. Namely one has 


$$
T \cdot \mathbf{z}_{\circ}=T \text { for } \mathbf{z}_{\circ}=(1,1, \ldots, 1) .
$$

Let $K$ denote a proper subgroup of $T$. The $T$-orbit $T \cdot \mathbf{z}_{\circ}$ is a disjoint union of $|T / K|$ orbits for the subgroup $K$. These correspond to the cosets of $K$ in $T$. As $\left(T, H_{\mathcal{W}}\right)$ is a Gelfand pair we have $|\mathcal{W} / T|=q^{n}$ and

$$
|\mathcal{W} / K| \geq q^{n}-1+|T / K|>q^{n} .
$$

So $\left(K, H_{\mathcal{W}}\right)$ fails to be a Gelfand pair by Proposition 4.2.

\section{References}

[BJR90] Chal Benson, Joe Jenkins, and Gail Ratcliff. On Gel'fand pairs associated with solvable Lie groups. Trans. Amer. Math. Soc., 321(1):85-116, 1990.

[Fol89] Gerald B. Folland. Harmonic analysis in phase space, volume 122 of Annals of Mathematics Studies. Princeton University Press, Princeton, NJ, 1989.

[Gér77] Paul Gérardin. Weil representations associated to finite fields. J. Algebra, 46(1):54-101, 1977.

[Hel84] Sigurdur Helgason. Groups and geometric analysis, volume 113 of Pure and Applied Mathematics. Academic Press Inc., Orlando, FL, 1984. Integral geometry, invariant differential operators, and spherical functions.

[How] Roger E. Howe. Invariant theory and duality for classical groups over finite fields, with applications to their singular representation theory. Unpublished manuscript.

[How73] Roger E. Howe. On the character of Weil's representation. Trans. Amer. Math. Soc., 177:287-298, 1973.

[Lep85] Horst Leptin. A new kind of eigenfunction expansions on groups. Pacific J. Math., 116(1):45-67, 1985.

[LN] Rudolf Lidl and Harald Niederreiter. Finite fields, volume 20 of Encyclopedia of Mathematics and its Applications. Second edition.

[Mac] I. G. Macdonald. Symmetric functions and Hall polynomials. Oxford Mathematical Monographs. Second edition.

[Neu02] Markus Neuhauser. An explicit construction of the metaplectic representation over a finite field. J. Lie Theory, 12(1):15-30, 2002.

[SA87] Jorge Soto-Andrade. Geometrical Gel'fand models, tensor quotients, and Weil representations. In The Arcata Conference on Representations of Finite Groups (Arcata, Calif., 1986), volume 47 of Proc. Sympos. Pure Math., pages 305-316. Amer. Math. Soc., Providence, RI, 1987.

[Shi80] Ken-ichi Shinoda. The characters of Weil representations associated to finite fields. J. Algebra, 66(1):251-280, 1980.

[Ter99] Audrey Terras. Fourier analysis on finite groups and applications, volume 43 of London Mathematical Society Student Texts. Cambridge University Press, Cambridge, 1999.

[Vin03] È. B. Vinberg. Commutative homogeneous spaces of Heisenberg type. $T r$. Mosk. Mat. Obs., 64:54-89, 2003.

[Yak06] Oksana Yakimova. Principal Gelfand pairs. Transform. Groups, 11(2):305335, 2006. 